
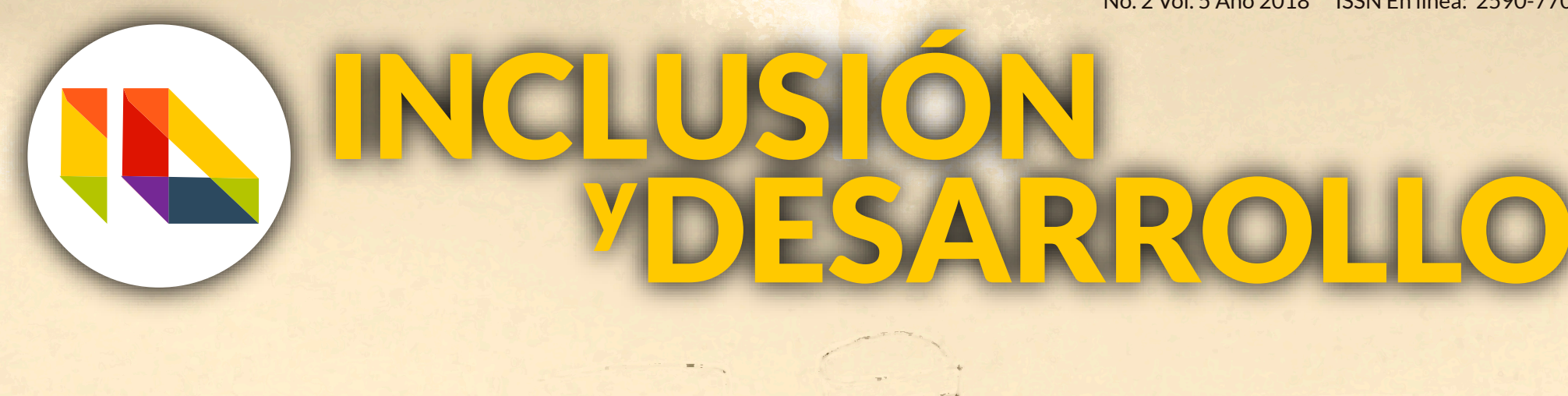

1

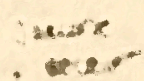

lite
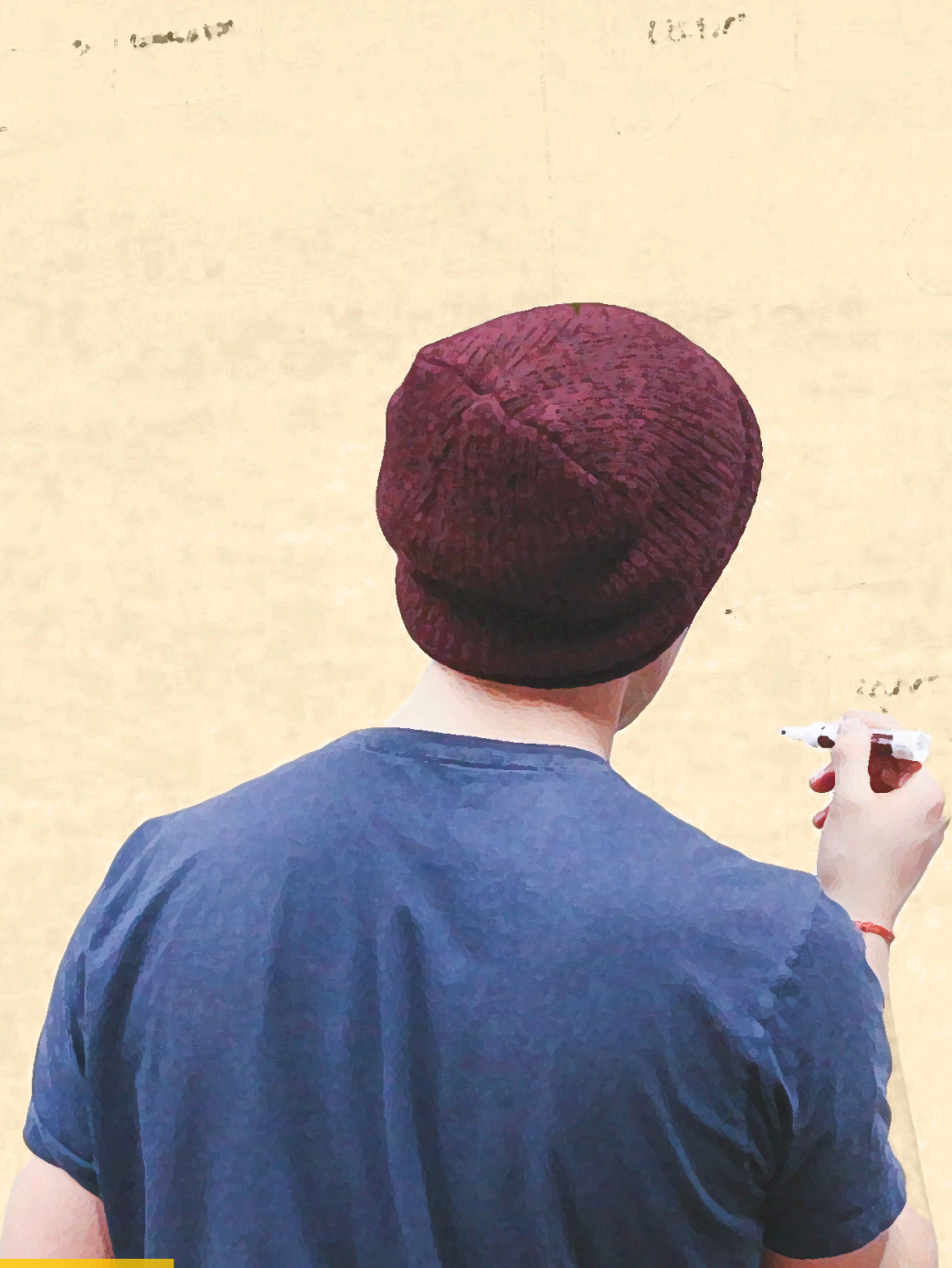

\title{
Estilos de enseñanza
}

y desarrollo de competencias

investigativas

en educación superior

Luis Augusto Cuellar, Esteban Muñoz Montenegro y Angel Zuley Pedraza Hurtado 


\section{ESTILOS DE ENSEÑANZA Y DESARROLLO DE COMPETENCIAS INVESTIGATIVAS EN EDUCACIÓN SUPERIOR}

\section{STYLES OF TEACHING AND DEVELOPMENT OF INVESTIGATIVE COMPETENCES IN HIGHER EDUCATION}

Luis Augusto Cuellar Icuellar31@yahoo.com Universidad de la Salle Bogotá-Colombia

Esteban Muñoz Montenegro mumon35@hotmail.com Universidad de la Salle Bogotá-Colombia

Angel Zuley Pedraza Hurtado apedraza@unisalle.edu.co Universidad de la Salle Bogotá-Colombia

Encuentre este artículo en

http://revistas.uniminuto.edu/

Fecha de recepcion: 12 de agosto de 2017

\section{Resumen}

Objetivo. Indagar por los estilos de enseñanza de algunos maestros y las competencias investigativas de sus respectivos estudiantes con el objeto de caracterizar los primeros e identificar las segundas, evidenciando cuáles estilos docentes promueven en mayor o menor grado las diversas competencias de investigación a través de su práctica en el aula.

Metodología. Se adoptó el tipo de estudio descriptivo y el diseño de investigación que se siguió fue el análisis transeccional descriptivo a fin de constatar las variables indicadas. Se parte de una contextualización respecto de la dinámica de la educación colombiana y en particular la de nivel superior, particularizando en el alma mater lasallista desde la perspectiva del SIUL como documento matriz de la investigación en su política y misión. Los referentes teóricos que dieron sustento al abordaje de la investigación, parten de los antecedentes investigativos de docentes universitarios colombianos como Hederich, Suárez y Oviedo.

Resultados. La conclusión general de la investigación aprecia que en cuanto a los estilos de enseñanza, cada docente comporta características de los cuatro estilos tomados como referencia: directivo, tutorial, planificador e investigador, pero que hay uno que en mayor grado de $\neg$ termina su práctica. En cuanto las competencias investigativas, dependen en gran medida de la manera como interactúe el docente con los estudiantes y del grado de énfasis que coloque en las actividades de aprendizaje a fin de conseguir favorecer dichas competencias. Todos los estilos pro $\neg$ mueven competencias investigativas. Los resultados pueden servir como punto de referencia para el fortalecimiento y consolidación de los semilleros de investigación en las universidades.

Originalidad. Los Estilos de Enseñanza constituyen un referente obligatorio a la hora de comprender su incidencia en la relación tripodal entre el conocimiento, el docente y el estudiante.

Palabras clave: estilos de enseñanza, competencias investigativas, relación pedagógica. 


\section{Abstract}

Objective. To Investigate the teaching styles of some teachers and the investigative skills of their students in order to characterize the former and identify the latter, demonstrating which teaching styles promote to a greater or lesser degree the various research competencies through their practice in Classroom.

Methodology. The type of descriptive study was adopted and the research design followed was the descriptive transectional analysis in order to verify the indicated variables. It starts from a contextualization regarding the dynamics of Colombian education, especially, that of higher education, particularly in the Lasallista alma mater from the perspective of SIUL as a matrix document of research in its policy and mission. The theoretical referents that gave support to the approach of the investigation, start from the investigative antecedents of Colombian university professors like Hederich, Suárez and Oviedo.

Results. The general conclusion of the research shows that in terms of teaching styles, each teacher has characteristics of the four styles taken as a reference: directive, tutorial, planner and researcher, but there is one that determines their practice to a greater degree. As far as the investigative competences, they depend to a large extent on the way the teacher interacts with the students and on the degree of emphasis that they place on the learning activities in order to be able to favor those competences. All styles promote research skills. The results can serve as a point of reference for the strengthening and consolidation of hotbed of research in universities.

Originality. The Teaching Styles constitute a mandatory reference when we try to understand their incidence in the tripodal relationship among knowledge, the teacher and the student.

Keywords: teaching styles, investigative skills, pedagogical relationship. 


\section{INTRODUCCIÓN}

U

na condición básica para que el país mejore en su desarrollo y crecimiento, es pasar de una sociedad de la información a una sociedad del conocimiento, como lo señala (Chaparro, 1999):

"En el nuevo milenio, cada individuo y cada organización construirá su capacidad de acción y, por tanto, su posición en la sociedad, mediante el conocimiento y la capacidad de generar nuevo conocimiento, que le permita adaptarse al ritmo veloz del cambio. Este es el rasgo central de las sociedades del conocimiento, o sociedades capaces de generar conocimientos acerca de su realidad y de su entorno, y capaces de utilizar dicho conocimiento para concebir y construir su futuro. De esta forma, el conocimiento se convierte no solo en instrumento para explicar y comprender la realidad, sino también en motor de desarrollo y en factor dinamizador del cambio social"

La anterior afirmación sirve de marco para ahondar en la reflexión y discusión acerca de la relación entre los conceptos de competencia e investigación y su relevancia en la formación de los estudiantes de educación básica, media y superior.

Así mismo, el concepto de competencia se liga al de la calidad, como el de la investigación y su aplicación, al desarrollo y avance de los países. Al relacionar los dos conceptos surge uno interesante, el de competencia investigativa, su importancia radica en que su apropiación tiene un efecto altamente positivo e impactante en desarrollo social y científico. Tal es así que, el Ministerio de Educación Nacional ha señalado como prioritario su discusión y apropiación, recuérdese que los exámenes de estado, tanto en la educación básica y media como en la superior están basados en la valoración por competencias. Además, esa valoración va más allá, tal como lo señala el plan decenal de educación 2006-2016, (Ministerio de Educación Nacional , 2006) "una tercera prioridad, se basa en la idea de formar el talento humano necesario para el desarrollo de la ciencia, la tecnología y la in $\neg$ novación."

Las anteriores reflexiones nos llevaron a investigar la relación entre las competencias investigativas y los estilos de enseñanza en algunos profesores de las facultades de Ingeniería y Educación en la Universidad de la Salle, teniendo como marco lo propuesto, por la Universidad en el diseño del Sistema de Investigación Universitario Lasallista - SIUL - a fin de pasar de una Universidad que enseña a una Universidad que aprende, y la cual señala como metas, entre otras:

"1.Generar al interior de la Universidad la cultura de la producción intelectual, científica y de innovación, fundada en la investigación.

2. Conformar, fortalecer y consolidar los grupos de investigación y el semillero de jóvenes investigadores.

3. Cualificar el ejercicio docente mediante la actividad investigativa con el fin de enriquecer el modelo pedagógico.”

(Universidad de la Salle, 2008).

A partir de estas consideraciones se generaron en el equipo investigador una serie de inquietudes que son apremiantes en la práctica pedagógica: ¿Cómo hacer para que los estudiantes se interesen en la investigación y que esta se constituya en elemento significativo de su formación? En esa medida, ¿de qué forma al acercamos a los estudiantes, potenciamos su ser de investigadores? ¿Cuál es la influencia de los estilos de enseñanza en los aprendizajes de los estudiantes? ¿El docente emplea o tiene diferentes estilos que utiliza en el aula? ¿Influye la personalidad del docente en su estilo de enseñanza? ¿Cómo perciben los estudiantes los estilos de enseñanza de sus maestros? ¿Los estilos de enseñanza favorecen o obstaculizan los aprendizajes de los estudiantes? Y la cuestión más definitiva para el presente estudio ¿Qué estilos de enseñanza favorecen el desarrollo de las competencias investigativas?

Es por esto que el propósito que persiguió la investigación fue: caracterizar los estilos de enseñanza que favorecen el desarrollo de competencias investigativas en los estudiantes universitarios, identificando los primeros y describiendo las segundas a partir de la triangulación de resultados de tres instrumentos: la observación directa no interviniente, la entrevista a docentes y dos cuestionarios a estudiantes (uno para indagar sobre estilos y otro para verificar el desarrollo de competencias). 


\section{ESTADO DEL ARTE Y ANTECEDENTES PRÁCTICOS}

Se relacionan a continuación algunos estudios teóricos e investigaciones que se han adelantado sobre el tema tanto en Colombia como en otros países latinoamericanos.

- Estudio exploratorio sobre las prácticas de enseñanza aprendizaje adecuadas de las /los profesores. Una mirada a partir de los estudiantes. Zoraida Ordoñez Pinzón. - Facultad de Trabajo Social. ULS 2007.

De este estudio destaca la caracterización que los estudiantes hacen de las prácticas de sus profesores, en particular lo atinente al aspecto de la investigación formativa. Los resultados refieren en particular al espacio académico dedicado al proceso de enseñanza-aprendizaje de la investigación, las intencionalidades de los profesores y las habilidades, intelectuales y actitudinales, que los estudiantes deben desarrollar para el logro de las mismas. Señala como estrategias metodológicas la reflexión vivencial y el taller, distinguiendo al docente como orientador y acompañante del proceso.

- Estrategia de formación en investigación en jóvenes universitarios. Yahemn Baeza Dáger Ms. Raimundo Abello Llanos Ph.D Dirección de Investigaciones y Proyectos Fundación Universidad del Norte. Foro: Competencias científicas en Educación Superior. Medellín, 2,3 y 4 de noviembre del 2005.

Presenta los resultados de la implementación de una estrategia para desarrollar competencias investigativas en estudiantes universitarios de cuarto a octavo semestre en la UNINORTE entre el año 2000 al 2005. Se destaca de la propuesta los tres momentos en que la desarrollaron: fundamentación Teórica, aproximación a lo científico y experiencia práctica.

- La renovación de los estilos pedagógicos: colectivos para la investigación y la acción en la universidad María Mercedes Callejas R., Martha Vitalia Corredor M. // En: Docencia universitaria. -- Bucaramanga: Universidad Industrial de Santander, Centro para el Desarrollo de la Docencia.-- Vol. 3, no.1 (May. 2002). -- p. 61-96 / Spa
Presenta una propuesta de formación de los profesores universitarios, orientada desde la investigación de los estilos pedagógicos. El proyecto inicia con la caracterización de los estilos a través de la reflexión de los profesores sobre sus concepciones y prácticas, la cual permite en una perspectiva crítica, identificar aspectos problemáticos de las mismas y desarrollar propuestas de investigación-acción que favorezcan la renovación de estos estilos. Es un buen referente contextual que puede aportar al diseño de los instrumentos a aplicar en la investigación.

- Los profesores en contextos de investigación e innovación. José Gregorio Rodríguez y Elsa Castañeda Bernal. En http://www.rinace. net/enlacerevistas.htm

Se tomó como punto de referencia sobre las investigaciones e innovaciones realizadas por los profesores en el contexto escolar y la relación docencia-investigación.

- Construcción de las competencias investigativas de los estudiantes del programa de Licenciatura en Educación Física y Recreación de la Universidad de Caldas en su trasegar por la asignatura investigación.

- José Enver Ayala. Tesis. Universidad de Manizales. Facultad de Educación y Psicología. Maestría en Educación. Manizales, 2006. correo.umanizalez.edu.co/tesis/MaeEdudo.

Es un proyecto de investigación afín, dado que el problema que plantean hace relación al desarrollo de competencias investigativas en estudiantes universitarios, particularmente a partir de la asignatura de Investigación. Sus resultados sirven como punto de referencia pues se enfocó a un programa de formación docente y varias de las preguntas problema apuntan en el mismo sentido al objetivo la de la presente propuesta.

- Enseñanza y aprendizaje en la Educación Superior

Dra. Diana L. Cruz M. (Abril 2001 - U. de Pto. Rico en Humacao) www.upr.clu.edu/

Presenta una amplia ilustración de estilos de enseñanza relacionados con estilos de aprendizaje sugiriendo aquellos que más posibilidades 
Tabla 1. Desarrollo De Competencias Investigativas Cuestionario Docente

\begin{tabular}{|c|c|c|c|c|}
\hline AFIRMACIONES & MUCHO & BASTANTE & POCO & NADA \\
\hline 1. Identificar y localizar fuentes de información & 5 & 5 & 1 & 0 \\
\hline 2. Saber seleccionar la información adecuada & 4 & 7 & 0 & 0 \\
\hline 3. Participar activamente en grupos de trabajo & 6 & 4 & 1 & 0 \\
\hline $\begin{array}{l}\text { 4. Trabajar en la consecución de } \\
\text { acuerdos y objetivos comunes }\end{array}$ & 1 & 9 & 1 & 0 \\
\hline $\begin{array}{l}\text { 5. Escuchar tomando en cuenta otras } \\
\text { apreciaciones y aportar constructivamente }\end{array}$ & 3 & 7 & 1 & 0 \\
\hline $\begin{array}{l}\text { 6. Participar en la organización y asignación } \\
\text { de tareas dentro del equipo }\end{array}$ & 3 & 6 & 2 & 0 \\
\hline $\begin{array}{l}\text { 7. Informar acerca de los avances y dificultades } \\
\text { en el desarrollo del trabajo investigativo }\end{array}$ & 5 & 5 & 1 & 0 \\
\hline $\begin{array}{l}\text { 8. Adaptar ideas con óptima redacción, citando } \\
\text { las correspondientes fuentes y referencias }\end{array}$ & 7 & 4 & 0 & 0 \\
\hline $\begin{array}{l}\text { 9. Presentar tablas gráficas y otros esquemas } \\
\text { para ilustración o sustentación de datos }\end{array}$ & 6 & 3 & 1 & 0 \\
\hline $\begin{array}{l}\text { 10. Formular problemas basado en conocimientos } \\
\text { previos y situaciones experienciales. }\end{array}$ & 3 & 7 & 1 & 0 \\
\hline $\begin{array}{l}\text { 11. Diseñar y aplicar instrumentos como } \\
\text { entrevistas, test encuestas, observaciones }\end{array}$ & 1 & 5 & 4 & 1 \\
\hline $\begin{array}{l}\text { 12. Diseño y elaboración de instrumentos de } \\
\text { recolección de información para una investigación. }\end{array}$ & 5 & 6 & 0 & 0 \\
\hline $\begin{array}{l}\text { 13. Evaluar el proceso y los resultados obtenidos } \\
\text { de acuerdo con los objetivos propuestos }\end{array}$ & 4 & 6 & 1 & 0 \\
\hline $\begin{array}{l}\text { 14. Innovar en el desarrollo de modelos } \\
\text { para resolver problemas }\end{array}$ & 4 & 5 & 2 & 0 \\
\hline $\begin{array}{l}\text { 15. Comprender la información técnica } \\
\text { y científica de una lectura }\end{array}$ & 6 & 3 & 2 & 0 \\
\hline $\begin{array}{l}\text { 16. Comunicar y realizar descripciones } \\
\text { del saber científico }\end{array}$ & 3 & 7 & 1 & 0 \\
\hline $\begin{array}{l}\text { 17. Llevar a cabo acciones de acuerdo con } \\
\text { un plan y un cronograma establecido }\end{array}$ & 3 & 5 & 3 & 0 \\
\hline $\begin{array}{l}\text { 18. Correlacionar información } \\
\text { proveniente de diferentes fuentes. }\end{array}$ & 6 & 5 & 0 & 0 \\
\hline
\end{tabular}




\begin{tabular}{|c|c|c|c|c|}
\hline AFIRMACIONES & MUCHO & BASTANTE & POCO & NADA \\
\hline $\begin{array}{l}\text { 19.D efinir clara y coherentemente } \\
\text { conceptos y las relaciones entre ellos. }\end{array}$ & 3 & 7 & 1 & 0 \\
\hline $\begin{array}{l}\text { 20. Comprender y encontrar sentido y significado a los } \\
\text { objetos de aná-lisis: textos, situaciones, problemas. }\end{array}$ & 6 & 4 & 1 & 0 \\
\hline $\begin{array}{l}\text { 21. Expresar las ideas en forma clara, precisa y oportuna } \\
\text { con propiedad tanto de manera oral como escrita }\end{array}$ & 5 & 5 & 1 & 0 \\
\hline 22. Formular preguntas relevantes & 3 & 5 & 3 & 0 \\
\hline $\begin{array}{l}\text { 23. Desarrollar la capacidad argumentativa } \\
\text { de manera que permita la sustentación de } \\
\text { una tesis, el diálogo y el debate racional }\end{array}$ & 7 & 3 & 1 & 0 \\
\hline $\begin{array}{l}\text { 24. Enunciar argumentos con propiedad, } \\
\text { tanto de manera oral como escrita }\end{array}$ & 7 & 4 & 0 & 0 \\
\hline TOTAL & 106 & 127 & 29 & 1 \\
\hline PORCENTAJE & $40.15 \%$ & $48.11 \%$ & $11.0 \%$ & $0.4 \%$ \\
\hline
\end{tabular}

tienen de ser adaptados a la educación superior. Plantea algunas recomendaciones, pero no aporta sobremanera a la práctica y al cometido del proyecto.

Algunas condiciones pedagógicas para la formación y el desarrollo de la investigación en la Universidad.

- Quiñones Cárdenas Jeremías - Vélez Álvarez Consuelo. En Revista Electrónica "Actualidades investigativas en educación” U. de Costa Rica revista@inie.ucr.ac.cr http://www.inie. ucr.ac.cr/

Desarrolla una propuesta innovadora para incentivar el ejercicio investigativo en el nivel superior de la Educación, a partir de requerimientos de tipo pedagógico. Puede servir como referente para identificar características de estilos de enseñanza que promuevan investigación en los estudiantes.

- Estudio de estilos de enseñanza en la Universidad. Estudio en tres carreras: Ciencias biomédicas, abogacía y comunicación social. Universidad Nacional de Mar del Plata.

Este estudio permitió identificar diferencias entre los estilos de enseñanza según las áreas disciplinares. Aporta un buen marco teórico y además como referencia para definir la unidad de observación y la muestra que puede tomar respecto a los profesores.

\section{MARCO TEÓRICO Y REFERENTES CONCEPTUALES}

En la estructura teórica se precisa el concepto de estilo y los estilos de enseñanza. De igual manera aborda los conceptos de competencias, investigación y competencias investigativas.

El concepto de estilo tiene origen en el arte, el profesor Cristian Hederich, investigador de la Universidad Pedagógica Nacional lo refiere como al conjunto de características que definen una tendencia estética identificable y distintiva, lo define igualmente como: "modalidades distintivas y claramente identificables en la expresión de una actividad humana." (Hederich, 2007) Se habla igualmente de estilo en cuanto a la moda, a la manera como se interpreta la música, al estilo de escribir, de hablar, de estilos de liderazgo, estilos de vida.

De acuerdo con Hederich, en la investigación sobre estilos de enseñanza hay dos líneas con propósitos y desarrollos teóricos diferentes: una psicológica y otra pedagógica. En la primera se 
conciben los estilos de enseñanza como una manifestación más del concepto de estilo cognitivo. En esta tradición se considera el estilo como "las formas preferidas de uso de las habilidades que se poseen. (Stemberg, 1997). Se concibe entonces el concepto de estilo de enseñanza en términos de las formas preferidas de enseñar del docente que resultan connaturales a su propio estilo cognitivo. Desde la tradición pedagógica, siguiendo con Hederich, "en vez de caracterizar la actividad concreta del profesor en el aula, intentan identificar concepciones o puntos de vista respecto a la labor educativa" (Hederich, 2007).

Desde el punto de vista del profesor Paulo Emilio Oviedo y su grupo investigador los estilos de enseñanza son considerados como: "La manera propia y particular como los docentes asumen la mediación pedagógica integralmente desde su saber, saber hacer y saber ser." (Oviedo y. o., 2005)

En la presente investigación se ha conceptualizado como estilo de enseñanza: "El conjunto de características propias, preferencias y habilidades del docente que este adecúa en el proceso de enseñar". De este modo quien enseña se define en razón a factores, comportamientos y actitudes que facilitan el aprendizaje de un individuo en una relación pedagógica.

De otra parte, la investigadora Cecilia Suárez y su grupo, partiendo de un estudio en la Universidad Sergio Arboleda, identificaron cuatro clases de estilos, señalando los respectivos indicadores (Suárez, 2003):

- Estilo directivo: es caracterizado por la disciplina, la severidad, la autoridad y las clases magistrales; el maestro es el principal responsable del proceso; el alumno es pasivo y responde a los requerimientos del docente. Aquí predomina el contenido del aprendizaje.

- El profesor establece reglas estrictas de comportamiento y las cumple.

- El docente se preocupa por la disciplina y exige silencio en clase

- El docente impone su forma de pensamiento.

- El docente no permite las discusiones en clase.

- El profesor domina todo el tiempo la clase

- El profesor no genera espacios de participación.

- El alumno siente temor de participar en clase.

- El profesor no permite preguntas.

- El alumno todo el tiempo escucha y toma apuntes.
- Estilo tutorial: aquí el maestro es un guía, un facilitador y mediador, que responde a los intereses y necesidades de los alumnos; el alumno, por su parte, es activo y autónomo. En este estilo se da un aprendizaje por descubrimiento, hay actitud dialogante, el aprendizaje parte de las necesidades e intereses del alumno, se maneja una conciencia crítica.

- El docente responde de manera individual y personalizada a los intereses del alumno.

- El docente sabe escuchar a los alumnos.

- El alumno desarrolla su proyecto personalizado de trabajo.

- El docente enseña a través de tutorías y orientaciones en los proyectos desarrollados por los alumnos, ya sea en forma individual o en pequeños grupos.

- Estilo planificador: el docente planea con anticipación sus actividades para apoyar a los alumnos tomando en cuenta las diversas clases de aprendizaje e inteligencias, y es un evaluador en el proceso; el alumno responde y es activo. Aquí predominan la multiplicidad de estrategias.

- El docente entrega con anticipación el plan de actividades.

- El docente evalúa en forma constante los temas desarrollados, para retroalimentar los procesos enseñanza-aprendizaje.

- El docente entrega oportunamente los materiales necesarios para el desarrollo de actividades.

- El docente explica con anticipación cómo se va a evaluar.

- El docente utiliza diferentes estrategias didácticas para responder a los objetivos y apoyar el aprendizaje

- El alumno planea y prepara con anticipación sus actividades.

- Estilo investigativo: es aquel que está orientado a la generación de nuevos conocimientos, con una mirada compleja e interdisciplinaria; el alumno es activo, exigente y está encaminado al desarrollo de proyectos científicos. Los problemas son claves para el desarrollo del conocimiento y del aprendizaje.

- El docente propone problemas interdisciplinarios que generan, de parte de los alumnos, proyectos para buscar soluciones.

- El docente orienta la enseñanza con enfoque interdisciplinario e investigativo.

- El docente estimula la generación de problemas por parte de sus alumnos y el desarrollo de proyectos. 
Tabla 2. Respuestas al cuestionario sobre estilos de enseñanza porcentaje

\begin{tabular}{|c|c|c|c|c|c|}
\hline AFIRMACIONES & $\begin{array}{c}\text { TOTAL } \\
\text { DESACUERDO }\end{array}$ & DESACUERDO & DEACUERDO & $\begin{array}{c}\text { TOTAL } \\
\text { DEACUERDO }\end{array}$ & ESTILO \\
\hline $\begin{array}{l}\text { 1. Las discusiones son poco frecuentes } \\
\text { en el desarrollo de la clase }\end{array}$ & $83 \%$ & $6 \%$ & $6 \%$ & $6 \%$ & DIRECTIVO \\
\hline $\begin{array}{l}\text { 2. El estudiante siente temor de hacer } \\
\text { aportes al desarrollo de la clase }\end{array}$ & $50 \%$ & $50 \%$ & $0 \%$ & $0 \%$ & DIRECTIVO \\
\hline $\begin{array}{l}\text { 3. El docente es un guía que responde a los } \\
\text { intereses y necesidades de los estudiantes }\end{array}$ & $5,6 \%$ & $0,0 \%$ & $22,2 \%$ & $72,2 \%$ & TUTORIAL \\
\hline $\begin{array}{l}\text { 4. El d ocente enseña con un enfoque } \\
\text { interdisciplinario e investigativo }\end{array}$ & $0,0 \%$ & $5,6 \%$ & $55,6 \%$ & $38,9 \%$ & INVESTIGADOR \\
\hline $\begin{array}{l}\text { 5. El docente señala reglas claras para } \\
\text { el desarrollo del espacio académico }\end{array}$ & $0,0 \%$ & $5,6 \%$ & $55,6 \%$ & $38,9 \%$ & DIRECTIVO \\
\hline $\begin{array}{l}\text { 6. El docente entrega oportunamente } \\
\text { los materiales necesarios para el } \\
\text { desarrollo de actividades. }\end{array}$ & $0,0 \%$ & $11,1 \%$ & $50,0 \%$ & $38,9 \%$ & PLANIFICADOR \\
\hline $\begin{array}{l}\text { 7. El estudiante escucha las exposiciones } \\
\text { del profesor tomando apuntes } \\
\text { acerca de lo que este argumenta }\end{array}$ & $0,0 \%$ & $5,6 \%$ & $55,6 \%$ & $38,9 \%$ & DIRECTIVO \\
\hline $\begin{array}{l}\text { 8. La inquietudes de los estudiantes } \\
\text { son escuchadas de manera } \\
\text { respetuosa por el docente }\end{array}$ & $0,0 \%$ & $0,0 \%$ & $5,6 \%$ & $94,4 \%$ & TUTORIAL \\
\hline $\begin{array}{l}\text { 9. El docente propone problemas } \\
\text { interdisciplinarios que generan, por } \\
\text { parte de los alumnos, proyectos } \\
\text { para buscar soluciones }\end{array}$ & $0,0 \%$ & $5,6 \%$ & $44,4 \%$ & $50,0 \%$ & INVESTIGADOR \\
\hline $\begin{array}{l}\text { 10. El docente enseña a través de } \\
\text { tutorías y orientaciones en los proyectos } \\
\text { desarrollados por los estudiantes }\end{array}$ & $0,0 \%$ & $11,8 \%$ & $52,9 \%$ & $35,3 \%$ & TUTORIAL \\
\hline $\begin{array}{l}\text { 11. El pensamiento expresado por } \\
\text { el docente es lo predominante } \\
\text { en las discusiones de clase }\end{array}$ & $0,0 \%$ & $44,4 \%$ & $27,8 \%$ & $27,8 \%$ & DIRECTIVO \\
\hline $\begin{array}{l}\text { 12. El docente orienta la enseñanza con } \\
\text { enfoque interdisciplinario e investigativo }\end{array}$ & $0,0 \%$ & $0,0 \%$ & $72,2 \%$ & $27,8 \%$ & INVESTIGADOR \\
\hline $\begin{array}{l}\text { 13. El docente evalúa en forma } \\
\text { constante los temas desarrollados, } \\
\text { para retroalimentar los procesos } \\
\text { enseñanza-aprendizaje }\end{array}$ & $0,0 \%$ & $5,6 \%$ & $55,6 \%$ & $38,9 \%$ & PLANIFICADOR \\
\hline
\end{tabular}




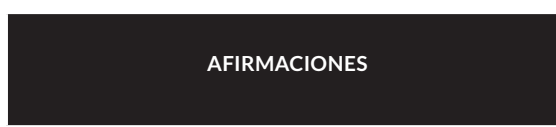

14. El docente estimula la generación de problemas por parte de sus alumnos y el desarrollo de proyectos

15. El docente explica con anticipación cómo se va evaluar

16. El docente utiliza diferentes estrategias didácticas para responder a los objetivos y apoyar el aprendizaje

17. El docente realiza tutorías de manera individual o en grupos durante el desarrollo del espacio académico

18. El docente estimula el pensamiento con sentido interdisciplinario e investigativo

19. El docente promueve el pensamiento crítico de sus estudiantes

20. El docente entrega con anticipación el plan de actividades

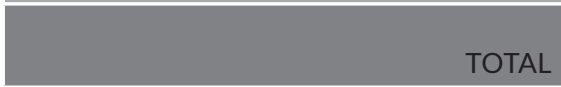

- Lo que se espera, fundamentalmente, es que el alumno, piense con sentido interdisciplinario e investigativo, desarrolle procesos de búsqueda y aplique procesos sistemáticos de investigación científica.

Para el abordaje de las competencias investigativas vale recordar que el término "competencia” fue acuñado por ChomskI (1965), en el contexto comunicacional al definirlas como "capacidades y disposiciones para la interpretación y la actuación, constituyendo una unidad conceptual y metodológica alejándose de un simple listado de actividades". Es decir, existe una estrecha relación entre competencias $\mathrm{y}$ actitudes.

En general las competencias investigativas se pueden entender como el conjunto de los conocimientos, las aptitudes profesionales y los conocimientos técnicos especializados que se aplican y dominan en un contexto específico.

\begin{tabular}{|c|c|c|c|}
\hline DESACUERDO & DEACUERDO & $\begin{array}{c}\text { TOTAL } \\
\text { DEACUERDO }\end{array}$ & ESTILO \\
\hline $0,0 \%$ & $72,2 \%$ & $22,2 \%$ & INVESTIGADOR \\
\hline $0,0 \%$ & $22,2 \%$ & $77,8 \%$ & PLANIFICADOR \\
\hline $11,1 \%$ & $38,9 \%$ & $50,0 \%$ & PLANIFICADOR \\
\hline $27,8 \%$ & $44,4 \%$ & $27,8 \%$ & TUTORIAL \\
\hline $0,0 \%$ & $50,0 \%$ & $50,0 \%$ & INVESTIGADOR \\
\hline $0,0 \%$ & $38,9 \%$ & $61,1 \%$ & TUTORIAL \\
\hline $0,0 \%$ & $33,3 \%$ & $66,7 \%$ & PLANIFICADOR \\
\hline $9 \%$ & $40 \%$ & $43 \%$ & \\
\hline
\end{tabular}

Este proceso de desarrollo de competencias debe entenderse como algo continuo: va desde los datos hasta la adquisición del conocimiento adecuado para que la acción sea eficaz y eficiente. Es así que para el propósito que se persigue, se construyó el concepto de competencia investigativa como: "El conjunto de habilidades, actitudes y conocimientos [recursos] desarrollados por la persona a partir de la mediación con el entorno sociocultural [estilos de enseñanza], que le permiten desempeñarse satisfactoriamente en las tareas propias de la investigación [contexto]". Conviene esta concepción con la de la investigadora mexicana María Cristina Torrado al afirmar que: "Ser competente, más que poseer un conocimiento, es saber utilizarlo de manera adecuada y flexible en nuevas situaciones".

Para operacionalizar las competencias investigativas tomamos como referencia el trabajo del grupo de Cuervo, Gómez y Rodríguez, docentes de la Fundación Universitaria Los Libertadores, 
quienes en su investigación para la Maestría en Docencia del año 2008, aplicaron la clasificación de las competencias investigativas en unidades y elementos de competencia así: (Cuervo et al, 2008).

\section{Buscar información}

- Identificar y localizar fuentes de información.

- Sabe cómo llegar a la información dentro de las fuentes.

- Selecciona críticamente la información más apropiada.

- Evalúa la calidad de la información obtenida.

\section{Trabajar en equipo}

- Se integra, participa y colabora activamente en las tareas del equipo.

- Se orienta a la consecución de acuerdos y objetivos comunes y se compromete con ellos.

- Toma en cuenta los puntos de vista de los demás y retroalimenta de forma constructiva.

- Colabora en la definición, organización y distribución de las tareas de grupo.

\section{Elaborar informes}

- Incluye en el informe: diseños, cálculos, ideas consideradas, precios, materiales, elementos utilizados, proceso para resolver el problema, dificultades que se presentaron.

- Parafrasea ideas, cita referencias correctamente y da los créditos correspondientes a las fuentes consultadas.

- Elabora gráficos, esquemas planos, modelos, listas para sustentar y hacer más claro el texto.

- Usa correctamente el idioma, expresa las ideas con claridad e incluye bibliografía completa de las fuentes consultadas.

\section{Resolver problemas}

- Relaciona y expresa experiencias y situaciones pasadas con el problema.

- Identifica y reconoce el problema dentro de una temática. Delimita los principales conceptos asociados con el problema a resolver.

- Inventa a partir de los conocimientos de que dispone, posibles respuestas, conceptos, ideas a modo de hipótesis. Identifica cual es la información necesaria y pertinente para resolver el problema.
- Diseña, ejecuta y evalúa planes de acción. Evalúa tanto el resultado obtenido como el proceso que se llevó a cabo para obtenerlo.

- Construye aparatos, equipos y dispositivos para resolver problemas.

\section{Interpretar textos científicos y técnicos}

- Comprende a cabalidad la información técnica y científica de una lectura.

- Identifica las ideas principales, las ideas de apoyo y las ideas de dominio.

- Define clara y coherentemente los conceptos y las relaciones entre ellos.

- Comprende y encuentra sentido y significado a los objetos de análisis: textos, situaciones, problemas.

\section{Argumentar y exponer ideas}

- Expresa las ideas en forma clara, precisa y oportuna y enuncia los argumentos con propiedad tanto de manera oral como escrita. Presenta argumentos relacionados con la tesis planteada, com $\neg$ puestos por una afirmación o conclusión y unas premisas o razo $\neg$ nes que los sustentan.

- Los conceptos básicos elaborados empleados en los argumentos no son contradictorios o confusos.

- Comunica y realiza descripciones y representaciones de su saber científico en forma correcta, verbalmente y por escrito.

\section{Metodología}

La presente investigación se fundamentó en un estudio descriptivo propuesto por Hernández, Fernández, Baptista (2001) "Los estudios descriptivos buscan especificar las propiedades importantes de personas, grupos, comunidades, o cualquier otro fenómeno que sea sometido a análisis" (2.001, p.60).

Este tipo de estudio fue elegido porque se ajustó a las características de la investigación planeada, "Los estudios descriptivos miden de manera más bien independiente los conceptos o variables a los que se refieren. Aunque, desde luego, pueden integrar las mediciones de cada una de dichas variables para decir cómo es y cómo se manifiesta el fenómeno de interés, su objetivo no es indicar cómo se relacionan las variables medidas". Hernández, Fernández, Baptista (2001) 
En este estudio cualitativo las variables que se describen de manera independiente son los estilos de enseñanza de los maestros y las competencias investigativas de los estudiantes.

En esta investigación se empleó un diseño transeccional descriptivo; según (Hernández et al. 2.001) los diseños transeccionales descriptivos tienen como objeto presentar un panorama del estado de una o más variables en uno o más grupos de personas en determinado momento.

En el caso del presente estudio el proceso se desarrolló de la siguiente manera:

1. Caracterización de los estilos de enseñanza de los docentes y competencias investigativas de los estudiantes mediante Observación y filmación de las clases.

2. Entrevista estructurada a cada uno de los docentes.

3. Aplicación de cuestionarios a los estudiantes sobre los estilos de enseñanza de los maestros y las competencias investigativas de los estudiantes.

Para obtener las evidencias que permitieran hacer el análisis cualitativo y la triangulación de las variables anteriormente señaladas se determina el proyecto de investigación con las siguientes características:

Unidades de observación: Seis (6) docentes: tres (3) de la Facultad de Ingeniería pertene- cientes al Programa de Diseño y Automatización electrónica y tres (3) de la Facultad de Educación adscritos al Programa de Licenciatura en Lengua castellana, inglés y francés; con sus grupos de estudiantes (de 20 estudiantes promedio) de sexto a noveno y de segundo y sexto semestre respectivamente.

Se observaron en total 9 sesiones de 2 horas cada una aproximadamente en el lapso de un mes, tiempo durante el cual también se realizaron las entrevistas a los docentes y se aplicaron los cuestionarios a los estudiantes.

Para construir y diseñar el instrumento de recolección de información se tomaron como referencia los indicadores señalados por Suárez (2005).

\section{ANÁLISIS Y RESULTADOS}

Para la realización del análisis de datos, se procuró que el proceso de digitación de la información recogida, fuera lo más precisa posible, no sólo en cuanto a la transcripción de los hechos, sino también a las actitudes y elementos no verbales que fueran de importancia para el estudio.

El análisis de la información se realizó teniendo en cuenta las operaciones analíticas descriptivas propuestas por Alfonso Torres (1996). A continuación, se mencionan; categorización y codificación, ordenación y clasificación, construcción de comparaciones.

Tabla 3. Estilos de Enseñanza con mayor tendencia Cuestionario Docente.

\begin{tabular}{l|c|c|}
\multicolumn{1}{c|}{ AFIRMACIONES } & TDEACUERDO & ESTILO \\
\hline $\begin{array}{l}\text { 9. El docente propone problemas interdisciplinarios } \\
\text { que generan, por parte de los alumnos, } \\
\text { proyectos para buscar soluciones }\end{array}$ & $50,0 \%$ & INVESTIGATIVO \\
\hline $\begin{array}{l}\text { 16. El docente utiliza diferentes estrategias didácticas } \\
\text { para responder a los objetivos y apoyar el aprendizaje }\end{array}$ & $50,0 \%$ & PLANIFICADOR \\
\hline $\begin{array}{l}\text { 18. El docente estimula el pensamiento con } \\
\text { sentido interdisciplinario e investigativo }\end{array}$ & INVESTIGATIVO \\
\hline $\begin{array}{l}\text { 19. El docente promueve el pensamiento } \\
\text { crítico de sus estudiantes }\end{array}$ & $50,0 \%$ & TUTORIAL \\
\hline 20. El docente entrega con anticipación el plan de actividades & $61,1 \%$ & PLANIFICADOR \\
\hline
\end{tabular}


Luego de transcribir las observaciones de clase y la entrevista, se buscó la manera de categorizar y codificar la información, para realizar esta actividad se tomaron en conjunto todos los estilos de enseñanza y se analizaron detenidamente para hallar las categorías que estuvieran transversalmente ligadas a todos los estilos, además se analizaron cada una de las competencias investigativas para construir unos indicadores generales de cada elemento de competencia, la codificación se realizó colocando letras mayúscula a cada párrafo.

En la tabla 1, se presentan los resultados que emergieron de la aplicación de los cuestionarios sobre competencias investigativas y estilos de enseñanza que fueron propuestos a los estudiantes de cada uno de los grupos con los cuales se desarrolló la investigación.

De la tabla 2 se observa: las afirmaciones, las inquietudes de los estudiantes son escuchadas de manera respetuosa por el docente y El docente explica con anticipación cómo se va evaluar son las de más alta puntuación; los estudiantes están totalmente de acuerdo en un $94.4 \%$ y $77.8 \%$ respectivamente, la primera característica corresponde al estilo tutorial, la segunda al estilo investigativo.

En la tabla 3 se pueden observar, las características correspondientes al estilo del docente observado, con su respectiva calificación.
$\mathrm{Al}$ sumar los porcentajes correspondientes: está totalmente de acuerdo y de acuerdo con las afirmaciones y relacionarlos con las características de cada estilo se encontró que en promedio, medido en frecuencias, las características más sobresalientes observadas por los estudiantes en el docente corresponden al estilo tutorial en un $58 \%$, seguido del planificador $54 . \%$, el investigativo con un $38 \%$ y el directivo con un $22 \%$, el resumen es como sigue, de igual forma se puede observar lo mencionado en figura $\mathrm{N}^{0} 1$.

\begin{tabular}{|c|c|c|c|}
\hline TUTORIAL & INVESTIGATIVO & PLANIFICADOR & DIRECTIVO \\
\hline $72,2 \%$ & $38,90 \%$ & $38,9 \%$ & $6,0 \%$ \\
\hline $94,4 \%$ & $50,00 \%$ & $38,9 \%$ & 0 \\
\hline $35,3 \%$ & $22,20 \%$ & $77,8 \%$ & $38,9 \%$ \\
\hline $27,8 \%$ & $50,00 \%$ & $50,0 \%$ & $38,9 \%$ \\
\hline $61,1 \%$ & $27,80 \%$ & $66,7 \%$ & $27,8 \%$ \\
\hline $58 \%$ & $38 \%$ & $54 \%$ & $22 \%$ \\
\hline
\end{tabular}

\section{CONCLUSIONES}

Después de realizar el estudio de los Estilos de Enseñanza de los docentes y las competencias investigativas de los estudiantes, mediante

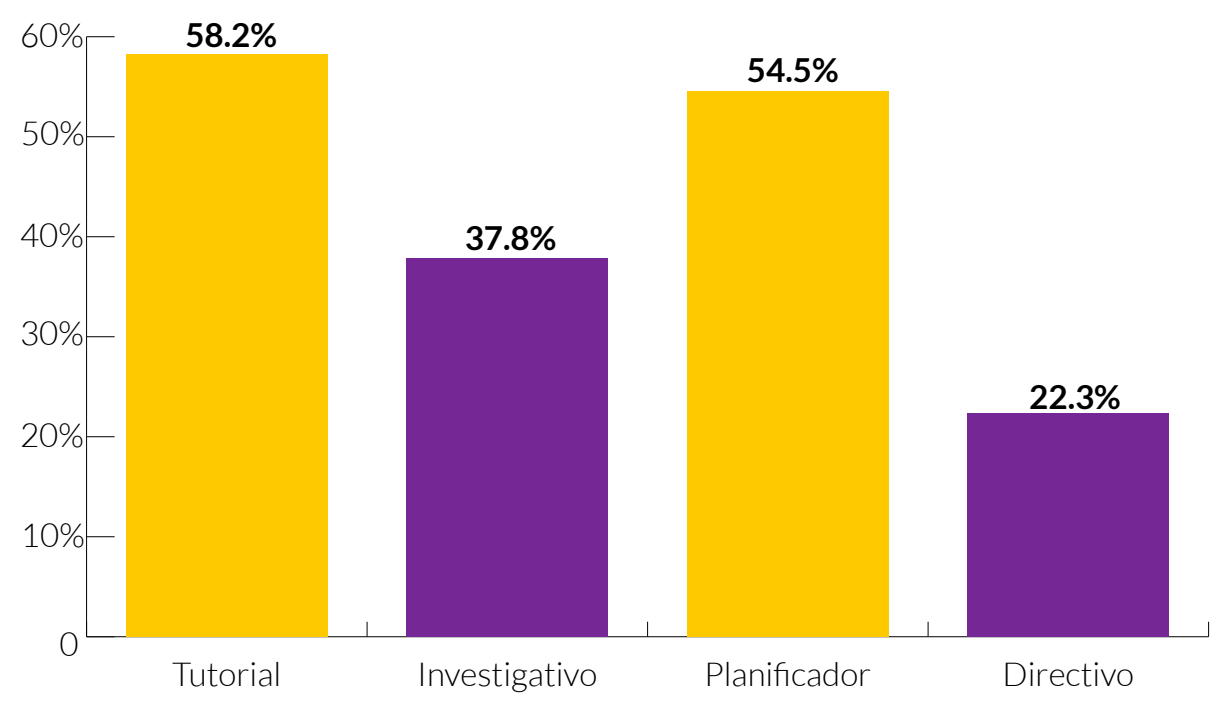

Figura 1. Promedio respuestas de cuestionario de Estilos de Enseñanza medido en porcentaje 
la recolección de la 58.237.8información, el análisis y su interpretación se pueden plantear las siguientes conclusiones:

Es de destacar que en esta investigación se corroboró lo que algunos investigadores, tales como Grasha y Bennet, observaron en sus propios estudios; que a pesar que predomina un estilo de enseñanza en cada docente, hay otros estilos que lo acompañan, estos se manifiestan en algunas fases del proceso enseñanza aprendizaje. También es necesario resaltar que todos los estilos de enseñanza favorecen el desarrollo de competencias investigativas, algunos estilos favorecen ciertas competencias particulares en mayor o menor escala. En ese sentido el estilo planificador tiende a favorecer más el desarrollo de la competencia investigativa de trabajo en equipo evidencia de esto es que al programar variadas actividades como: seguimiento grupal de indicaciones, ejecución nutrida de técnicas participativas, organización de grupos, trabajo independiente de los estudiantes, delegación de actividades por parte de los mismos estudiantes, trabajo colectivo integrado, presencia destacada de líderes, búsqueda del éxito en las actividades grupales, se promueven las habilidades para la labor de conjunto. El estilo investigador promueve significativamente la búsqueda de alternativas de solución, fomentando en los jóvenes estudiantes la inquietud por acercarse de manera más profunda al conocimiento de las causas del problema y a la búsqueda sistemática de respuestas, todos éstos rasgos de competencias investigativas. $E l$ estilo tutorial al caracterizarse por la orientación del proceso de aprendizaje promueve el aporte constructivo, participar en la organización y asignación de tareas dentro del equipo, definición de conceptos y comprensión de objetos de análisis, como también el desarrollo de la capacidad argumentativa y la formulación de problemas, todos estos elementos de competencia investigativa.

Este panorama permite constatar que el docente, sea cual sea el estilo que le caracteriza, sin tener la intención sistemática de promover en los estudiantes una habilidad o competencia investigativa específica, estas se desarrollan y manifiestan de modo rutinario en el desempeño académico de los mismos. A través de la investigación se constató la manifestación de algunas de las competencias investigativas en los estudiantes y esto se puede constituir en un punto de referencia para el fortalecimiento y consolidación de los semilleros de investigación en la universidad. Se avanzaría con más celeridad si se planteara claramente la manera como todos los docentes pueden encaminarse a alcanzar la meta de promover jóvenes investigadores.

Desde los primeros semestres se debería posibilitar el proceso investigativo, y en cada asignatura potenciar intensamente algunas competencias investigativas. Es claro que los estudiantes sí manifiestan unas competencias para la investigación, lo que en realidad hace falta es darles el espacio para el desarrollo especifico de una investigación en particular, es decir se tienen todos los implementos para armar un edificio, pero no se ha previsto la intención de hacerlo. Debe existir una decisión contundente de todo el equipo docente y directivo por encaminar las competencias investigativas de los estudiantes hacia la resolución sistemática de las diversas problemáticas que les rodean 


\section{Referencias bibliográficas}

Allport, W. (2004). Personality: a psycological interpretation. Recuperado el 10 de junio de 2008, de Revista Creando. UN de Colombia Sede Manizales: www.manizales.unal.edu.co/modules/unrev_creando/documentos/EstilosCognitivos.pdf

Ayala, J. (2006). Tesis de grado. Recuperado el 28 de abril de 2008, de Universidad de Manizales: www.umanizales.edu.co

Best, J. (1982). Cómo investigar en Educación. Madrid: Morata.

Bomnín, J. (2001). Revista Vitral $N^{o}$ 43. Recuperado el 9 de mayo de 2008, de Universidad Sergio Arboleda: http://www. usa.edu.co/civilizar/invedusa/marco.htm

Callejas, M. (2003). Grupos de investigación. Recuperado el 27 de mayo de 2008, de Universidad Pedagógica Nacional: www.pedagogica.edu.co

Callejas, M. (2002). La renovación de los estilos pedagógicos: colectivos para la investigación y la acción en la universidad . Docencia Universitaria UIS, 61-96.

Chaparro, F. (1999). De la Sociedad de la Información a la Sociedad del Conocimiento . En H. G. BUENDÍA, ¿Para dónde va Colombia? (págs. 252-258). Bogotá: Tercer Mundo editores .

Consejo Directivo. (2007). PEUL. Proyecto Educativo Universitario Lasllista. Bogotá, D.C., Colombia.

Cruz, D. (2001). Enseñanza y aprendizaje en la Educación Superior. Recuperado el 28 de abril de 2008, de Universidad de Pto. Rico en Humacao: www.upr.clu.edu

Cuervo, F. (2008). La resolución de problemas como estrategia didáctica en el desarrollo de elementos de la competencia investigativa en la formación de Ingenieros. Bogotá, D.C.: Universidad de La Salle.

Dirks, J. (1997). Estrategias para crear un ambiente oportuno para el aprendizaje. Recuperado el 9 de mayo de 2008, de Universidad Sergio Arboleda: www.usa.edu.co/civilizar/invedusa/marco/htm

Grasha, A. (1996-2002). Teaching with style . Recuperado el 2 de mayo de 2008, de Doc. Acreditación. Universidad de La Salle: http://www.lasalle.edu.co

Hederich, C. (2007). El estilo de enseñanza, un concepto en búsqueda de precisión. Pedagogía y Saberes, 31-40.

Lorenzo, A. (2005). Estilos de aprendizaje y enseñanzza. México: Trillas.

Ministerio de Educación NacionaL . (15 de noviembre de 2006). mineducacion.gov.co. Recuperado el 20 de agosto de 2009, de mineducacion.gov.co: PLAN\%20DECENAL\%20DE\%20EDUCACI\%80\%Ao\%A6\%D3N.\%202006-2016.\%20 CARTILLA.pdf

Ministerio de Educación Nacional. (2007). Marco de referencia para política pública sobre educación superior por ciclos y competencias. Recuperado el 2 de mayo de 2008, de MEN: www.mineducacion.gov.co

Navarro, R. (2004). El concepto de enseñanza aprendizaje. Recuperado el 19 de mayo de 2008, de Red Científica: www. redcientífica.com

Ordoñez, P. (2007). Estudio exploratorio sobre las practicas de enseñanza aprendizaje adecuadas de las /los docentes. Una mirada a partir de los estudiantes. Revista de Investigaciones UNISALLE.

Oviedo, P. (2007). Los paradigmas de investigación y la formación del docente universitario como investigador. Bogotá, D.C., Colombia.

Oviedo, P. (2005). Los estilos pedagógicos y la investigación acción. Pedagogía y saberes, 39-44.

Stenhouse, L. (1998). Investigación y desarrollo del currículo. Madrid: Morata.

Suárez, C. (2003). Informe de avance de la investigación de los estilos pedagógicos y su impacto en el aprendizaje de los alumnos. Recuperado el 27 de mayo de 2008, de Revista Civilizar Universidad Sergio Arboleda: www.usergioarboleda. edu.co

Torres, A. (1996). Estrategias y Técnicas de Investigación Cualitativa. Bogotá: UNISUR.

Universidad de Costa Rica. (2007). Revista Actualidades Investigativas en Educación. Recuperado el 15 de abril de 2008, de Universidad de Costa Rica: www.inie.ucr.ac.cr 
Universidad de la salle . (2008). Sistema de Investigación Universitario Lasallista SIUL. Bogotá: Oficina de medios Audiovisuales y Publicaciones .

Universidad de la Salle. (2007). Informe Final de Acreditación. Bogotá, D.C. 
Revista incluida en los siguientes agregadores de contenidos

Revista incluida en los siguientes directorios

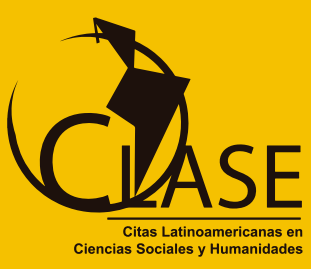

แlbiblat

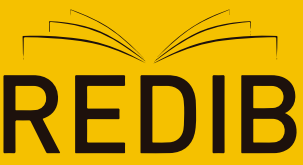

Red Iberoamericana

de Innovación y Conocimiento Científico

\section{Google Académico}

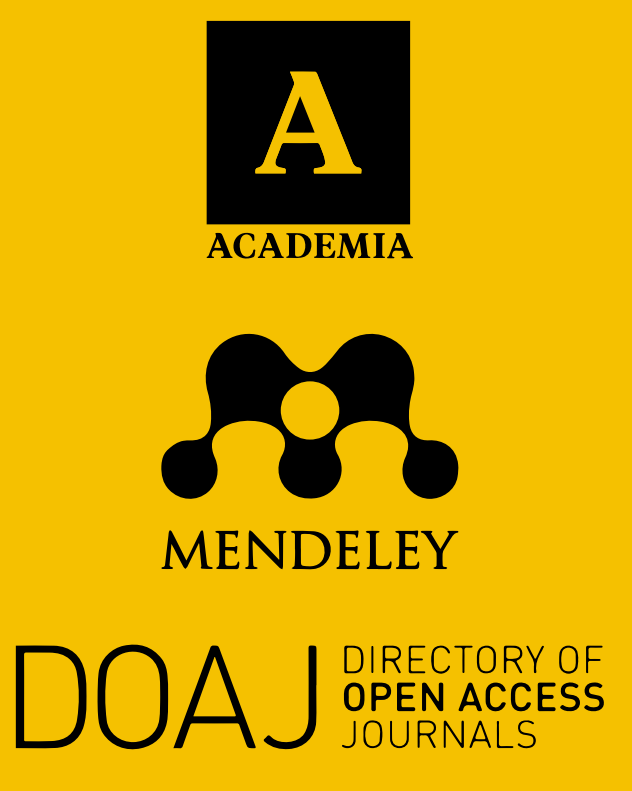

Revista incluida en la siguiente red social

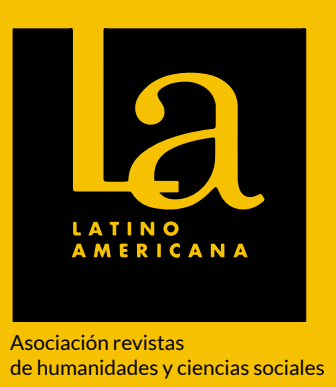



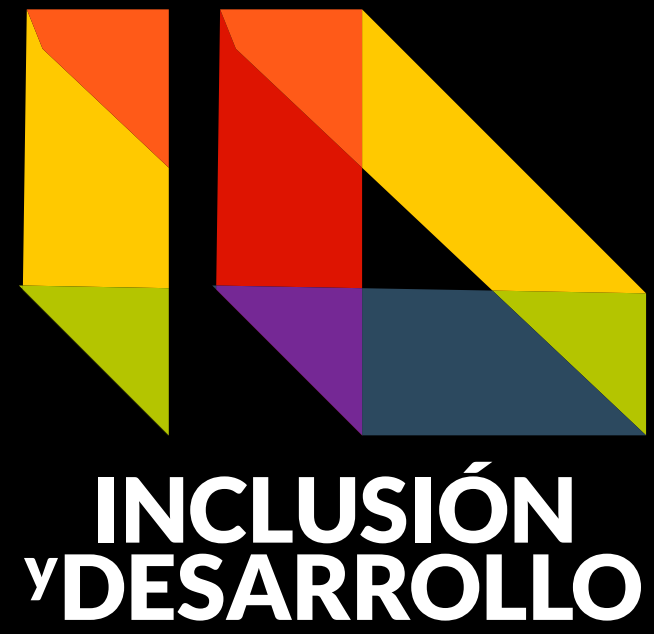

No. 2 Vol. 5 Año 2018 ISSN En línea: 2590-7700

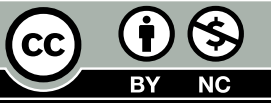

